\title{
Association between Circulatory and Plaque Resistin Levels with Carotid Plaque Instability and Ischemic Stroke Events
}

\author{
Ivana Jurin, MD,${ }^{1}$ Frane Paić, $\mathrm{PhD},{ }^{2,3}$ Stela Bulimbašić, $\mathrm{MD}, \mathrm{PhD},{ }^{4}$ Igor Rudež, $\mathrm{MD}, \mathrm{PhD}, 3$ \\ Lovorka Đerek, MSc, ${ }^{6}$ Hrvoje Jurin, MD,${ }^{7}$ Aleksandar Knežević, MD, PhD,${ }^{8}$ Boris Starčević, MD, PhD, ${ }^{1}$ \\ Marko Ajduk, MD, $\mathrm{PhD}^{9}$ \\ ${ }^{1}$ Department of Cardiovascular Diseases, University Hospital Dubrava, Avenija Gojka Šuška 6, 10000 Zagreb, Croatia; \\ ${ }^{2}$ Laboratory for Epigenetics and Molecular medicine, Department of Biology and Medical Genetics, School of Medicine, \\ University of Zagreb, Šalata 3, Zagreb, Croatia; ${ }^{3}$ Laboratory for Cardiovascular Biomedicine, Department of Cardiac and \\ Transplantation Surgery, University Hospital Dubrava, Avenija Gojka Šuška 6, 10000 Zagreb, Croatia; ${ }^{4}$ Department of \\ Pathology and Cytology, University Hospital Centre Zagreb, Avenija Gojka Šuška 6, 10000 Zagreb, Croatia; ${ }^{5}$ Department \\ of Cardiac and Transplantation Surgery, University Hospital Dubrava, Avenija Gojka Šška 6, 10000 Zagreb, Croatia; \\ ${ }^{6}$ Clinical Institute for Chemistry, University Hospital Centre "Sestre Milosrdnice", Vinogradska cesta 29, 10000 Zagreb, \\ Croatia; ${ }^{7}$ Department of Cardiovascular Diseases, University Hospital Centre Zagreb, Kišpatićeva 12, 10000 Zagreb, Croatia; \\ ${ }^{8}$ Department of Cardiology, General Hospital Zadar, Bože Peričića 5, 23000 Zadar, Croatia; ${ }^{9}$ Department of Vascular Surgery, \\ University Hospital Dubrava, Avenija Gojka Šuška 6, 10000 Zagreb, Croatia
}

\section{ABSTRACT}

Background: Carotid artery stenosis is one of the main causes of ischemic stroke. The risk of ischemic stroke increases with the degree of carotid stenosis and plaque vulnerability. The aim of this study was to investigate the association of circulating and plaque resistin levels with plaque vulnerability and ischemic stroke events in patients with moderate- to high-grade carotid artery stenosis.

Methods: 40 patients with ischemic stroke events and 38 neurologically asymptomatic patients scheduled for carotid endarterectomy were recruited for this study. Fasting blood samples for laboratory analysis were collected preoperatively and serum resistin levels were measured by enzyme-linked immunosorbent assays. Carotid endarterectomy specimens were analyzed according to the gold-standard procedure of histological classification. Plaque resistin expression was determined by standard immunohistochemical procedure.

Results: Serum resistin levels and resistin plaque expression were found to be significantly higher in subjects with unstable carotid plaque $(P<.001)$ while significantly higher serum resistin levels were also present in patients with ischemic stroke events $(P<.001)$. In univariate stepwise logistic regression analysis, higher serum resistin levels were significantly associated with plaque instability (OR 2.223, 95\% CI 1.488-3.320, $P<.0001)$ and ischemic stroke events (OR $1.237,95 \%$ CI $1.079-1.420, P=.002)$. There was also a significant association between higher serum and plaque resistin expression (OR 1.663, 95\% CI1.332-2.077, $P<.0001$ ). These associations remained significant in all models of multivariate

Received fune 20, 2018; accepted October 3, 2018.

Correspondence: Frane Paić, Assistant Professor, PhD, Laboratory for Epigenetics and Molecular Medicine, Department of Biology and Medical Genetics, School of Medicine, University of Zagreb, Salata 3, 10000 Zagreb, Croatia.+385114566804; fax: +3854590199 (e-mail:fpaic@mef.hr). logistic regression analysis. High serum and plaque resistin levels were also significantly associated with specific histological features of plaque instability.

Conclusion: The results suggests that serum resistin levels may be used as a potential biomarker of plaque vulnerability and ischemic stroke events in patients with moderate- to high-grade carotid artery stenosis and highlight the possible relationship that plaque resistin expression has with histological features of plaque vulnerability.

\section{INTRODUCTION}

Ischemic stroke is one of the leading causes of adult mortality, morbidity, and long-term disability in developed countries, with $10 \%-15 \%$ of cases caused by carotid artery stenosis [Mukherjee 2000; Flaherty 2013; Faxon 2004]. Both symptomatic and asymptomatic patients with higher degree of carotid artery stenosis (CAS) carry a greater risk for ischemic stroke development [Nighoghossian 2005]. However, plaque vulnerability cannot be determined solely by its size and the degree of lumen obstruction. Still, histological features associated with plaque instability leading to its rupture and thromboembolism formation are clearly visible only upon the carotid endarterectomy (CEA). Thus, the conventional clinical and imaging markers (carotid artery intima/media thickness, microembolic signals on transcranial Doppler, status of fibrous cap, lipid core and intraplaque hemorrhage and the degree of overall plaque inflammation, calcification, erosion and thrombosis) should be complemented by the new noninvasive biological biomarkers underlying the key pathophysiologic mechanisms on a cellular and molecular level [Nighoghossian 2005]. As such, they will more accurately identify plaque instability and patients nearfuture ischemic stroke risk.

High resistin serum levels and a proinflammatory cytokine that is mainly produced by monocytes and macrophages in humans were associated with an increased incidence of acute 
Table 1. Patients Demographic and Clinical Data Stratified According to Ischemic Stroke and Plaque Stability

\begin{tabular}{|c|c|c|c|c|c|c|c|}
\hline Male sex, n (\%) & $48(61.5)$ & $28(70.0)$ & $20(52.6)$ & .115 & $11(44.0)$ & $37(69.8)$ & $.029 * *$ \\
\hline BMI, $\mathrm{kg} / \mathrm{m}^{2}$ & $28.10 \pm 3.40$ & $27.80 \pm 2.87$ & $28.42 \pm 3.90$ & .422 & $28.47 \pm 3.70$ & $27.93 \pm 3.28$ & .520 \\
\hline Systolic BP, mm Hg & $135(130-140)$ & $140(130-140)$ & $130(123.75-140)$ & .065 & $140(130-142.5)$ & $130(130-140)$ & .426 \\
\hline Diastolic BP mm Hg & $80(80-90)$ & $80(80-90)$ & $82.5(80-90)$ & .817 & $90(80-90)$ & $80(80-90)$ & .795 \\
\hline Ischemic stroke, n (\%) & - & - & - & - & $5(20.0)$ & $35(66.0)$ & $.0002 * *$ \\
\hline Plaque stability, unstable, n (\%) & $53(67.9)$ & $35(87.5)$ & $18(47.4)$ & $.0002 *$ & - & - & - \\
\hline CAD, n (\%) & $18(23.1)$ & $7(17.5)$ & $11(28.9)$ & .230 & $6(24.0)$ & $12(22.6)$ & .894 \\
\hline PAD, n (\%) & $25(32.1)$ & $10(25.0)$ & $15(39.5)$ & .171 & $7(28.0)$ & $18(34.0)$ & .598 \\
\hline Aortic stenosis, $\mathrm{n}(\%)$ & $12(15.4)$ & $7(17.5)$ & $5(13.2)$ & .595 & $3(12.0)$ & $9(17.0)$ & .569 \\
\hline Pulmonary hypertension, $\mathrm{n}(\%)$ & $19(24.4)$ & $9(22.5)$ & $10(26.3)$ & .695 & $7(28.0)$ & $12(22.6)$ & .607 \\
\hline Diabetes, n (\%) & $45(57.7)$ & $22(57.9)$ & $23(57.5)$ & .972 & $15(60.0)$ & $30(56.6)$ & .777 \\
\hline Hyperlipidemia, n (\%) & $69(88.5)$ & $36(90.0)$ & $33(86.8)$ & .663 & $22(88.0)$ & $47(88.7)$ & .930 \\
\hline Beta blockers, n (\%) & $31(39.7)$ & $14(35.0)$ & $17(44.7)$ & .380 & $12(48.0)$ & $19(35.8)$ & .306 \\
\hline Antihyperglycemic, n (\%) & $18(23.1)$ & $11(27.5)$ & $7(18.4)$ & .341 & $5(20.0)$ & $13(24.5)$ & .658 \\
\hline Insulin injections, $\mathrm{n}(\%)$ & $11(14.1)$ & $6(15.0)$ & $5(13.2)$ & .815 & $4(16.0)$ & $7(13.2)$ & .741 \\
\hline Triglycerides, $\mathrm{mmol} / \mathrm{L}$ & $1.59(1.15-2.11)$ & $1.60(1.35-2.02)$ & $1.52(1.52-2.17)$ & .436 & $1.63(1.21-2.31)$ & $1.55(1.15-1.98)$ & .416 \\
\hline hsCRP, mg/L & $2.45(1.18-4.43)$ & $2.00(1.10-3.56)$ & $2.90(1.20-5.28)$ & .310 & $2.80(1.00-5.45)$ & $2.10(1.15-4.45)$ & .503 \\
\hline Fibrinogen, $g / L$ & $4.21 \pm 0.95$ & $4.05 \pm 0.86$ & $4.39 \pm 1.02$ & .108 & $4.28 \pm 0.96$ & $4.18 \pm 0.95$ & .659 \\
\hline $\mathrm{HbA} 1 \mathrm{C}, \%$ & $6.30(5.70-7.23)$ & $6.25(5.70-7.58)$ & $6.40(5.70-7.16)$ & .753 & $6.30(5.5-7.25)$ & $6.30(5.7-7.3)$ & .680 \\
\hline Leukocytes & $7.60(6.13-9.03)$ & $7.65(6.05-9.575)$ & $7.60(6.13-8.80)$ & .764 & $7.300(5.80-9.15)$ & 7.800 (6.40-9.05) & .392 \\
\hline Lymphocytes & $2.10(1.50-2.53)$ & $2.20(1.43-2.50)$ & $2.10(1.78-2.60)$ & .892 & $2.10(1.80-2.70)$ & $2.10(1.45-2.50)$ & .450 \\
\hline Neutrophils & $4.65(3.40-6.33)$ & $4.70(3.33-6.78)$ & $4.45(3.48-5.90)$ & .515 & $4.40(3.25-5.50)$ & $4.70(3.55-6.75)$ & .198 \\
\hline Resistin, ng/mL & $\begin{array}{c}6.643 \\
(4.413-10.213)\end{array}$ & $\begin{array}{c}8.789 \\
(5.933-11.105)\end{array}$ & $\begin{array}{c}4.683 \\
(3.876-8.487)\end{array}$ & $.0003 *$ & $\begin{array}{c}4.372 \\
(2.780-4.921)\end{array}$ & $\begin{array}{c}8.952 \\
(5.927-11.353)\end{array}$ & $<.0001 * *$ \\
\hline
\end{tabular}

Normally distributed data values are represented by mean \pm SD; Non-normally distributed data values are represent by median (interquartile range). ACE indicates angiotensin-converting-enzyme; BMl, body mass index; BP, blood pressure; CAD, coronary artery disease; CAS, coronary artery stenosis; HbA1c, glycated hemoglobin; HDL, high-density lipoprotein; hsCRP, high sensitivity C-reactive protein; LDL, low-density lipoprotein; T2DM, type 2 diabetes mellitus; WC, waist circumference; *P indicates significance between patients with ischemic stroke and patients without cerebrovascular symptomatology. **P indicates significance between patients with stable and patients with unstable plaques. 
Table 2. Non-Adjusted and Adjusted Spearman Correlation Coefficients between Plasma Resistin Levels and Hematological, Metabolic, and Cardiovascular Risk Factors in Whole Patient Group and Patient Groups with Ischemic Stroke and Unstable Plaque Phenotype

\begin{tabular}{|c|c|c|c|c|c|c|c|c|c|c|c|c|}
\hline & rS & $P$ & rS & $P$ & rS & $P$ & rS & $P$ & rS & $P$ & rS & $P$ \\
\hline WC & 0.197 & .083 & 0.274 & $.017^{*}$ & 0.470 & $.002 *$ & 0.485 & $.002 *$ & 0.504 & $<.001 *$ & 0.550 & $<.001 *$ \\
\hline $\mathrm{HDL}$ & -0.104 & .367 & -0.014 & .903 & -0.293 & .067 & -0.227 & .177 & -0.165 & .237 & -0.177 & .418 \\
\hline Triglyceride & 0.171 & .135 & 0.040 & .734 & 0.395 & $.012 *$ & 0.225 & .181 & 0.465 & $<.001 *$ & 0.305 & $.032 *$ \\
\hline Total Cholesterol & 0.111 & .335 & 0.175 & .132 & 0.170 & .295 & 0.093 & .585 & 0.146 & 0.298 & 0.137 & .342 \\
\hline Leukocytes & 0.342 & $.002 *$ & 0.287 & $.012 *$ & 0.463 & $.003 *$ & 0.315 & .058 & 0.469 & $<.001 *$ & 0.379 & $.007 *$ \\
\hline Neutrophils & 0.350 & $.002 *$ & 0.270 & $.019 *$ & 0.451 & $.004 *$ & 0.242 & .149 & 0.420 & $.002 *$ & 0.317 & $.025 *$ \\
\hline hsCRP & 0.059 & .609 & -0.065 & .555 & 0.275 & .086 & -0.055 & .747 & 0.170 & .224 & 0.134 & .353 \\
\hline
\end{tabular}

BP indicates blood pressure; HDL, high density lipoprotein; hsCRP, high sensitivity C-reactive protein; HbA1c, glycated hemoglobin; LDL, low density lipoprotein; rs, Spearman correlation coefficient; WC, waist circumference. *Significant $P$ values for non-adjusted and adjusted Spearman correlation with plasma resistin levels. Adjustments were performed for age, sex, and body mass index.

Table 3. Non-Adjusted and Adjusted Spearman Correlation Coefficients between Plasma Resistin Levels and Hematological, Metabolic, and Cardiovascular Risk Factors in Patients with AHA Plaque Type VI, T2DM, and Family History of Cerebrovascular Symptomatology

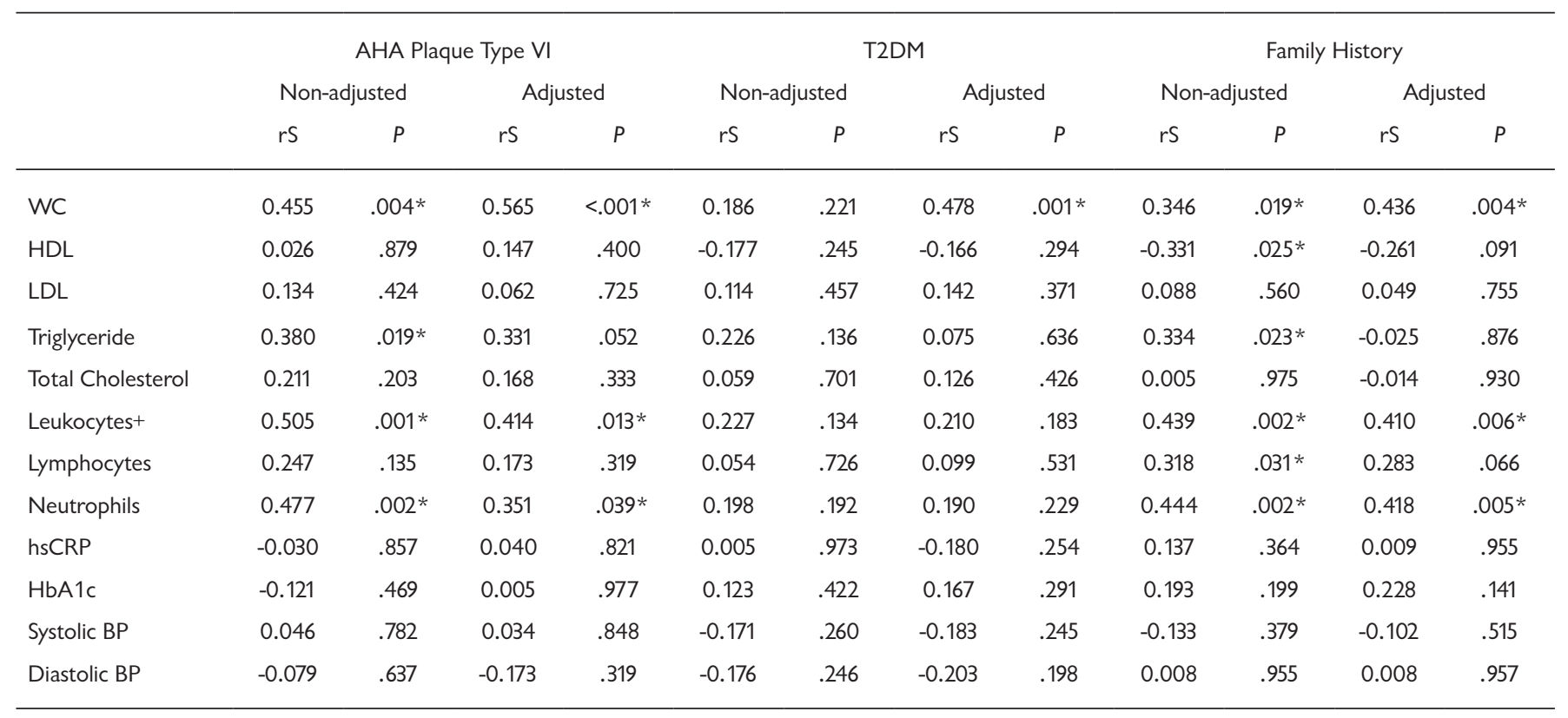

AHA indicates American Heart Association; BP, blood pressure; HDL, high density lipoprotein; hsCRP, high sensitivity C-reactive protein; HbA1c, glycated hemoglobin; LDL, low density lipoprotein; rs, Spearman correlation coefficient; T2DM, type 2 diabetes mellitus; WC, waist circumference. *Significant P values for non-adjusted and adjusted Spearman correlation with plasma resistin levels. Adjustments were performed for age, sex, and body mass index. 
Table 4. Overall Multiple Linear Regression Analysis between Plasma Resistin Levels and Hematological, Metabolic, and Cardiovascular Risk Factors + Age, Sex, and Body Mass Index

\begin{tabular}{|c|c|c|c|c|c|c|c|c|c|c|c|c|}
\hline & $\beta$ & $P$ & $\beta$ & $P$ & $\beta$ & $P$ & $\beta$ & $P$ & $\beta$ & $P$ & $\beta$ & $P$ \\
\hline WC & 0.216 & $.046 *$ & 0.509 & $.001 *$ & 0.727 & $<0.001$ & 0.704 & $.001 *$ & 0.847 & $<.001$ & 0.331 & $.014 *$ \\
\hline $\mathrm{HDL}$ & 0.100 & .392 & -0.206 & .147 & 0.040 & .736 & 0.238 & .075 & -0.076 & .580 & -0.088 & .550 \\
\hline LDL & 0.139 & .184 & -0.003 & .982 & 0.015 & .891 & 0.025 & .847 & 0.146 & .281 & -0.050 & .695 \\
\hline Cholesterol & 0.155 & .145 & -0.038 & .800 & 0.035 & .752 & 0.107 & .414 & 0.113 & .405 & -0.087 & .497 \\
\hline Leukocytes & 0.231 & $.036^{*}$ & 0.281 & .052 & 0.284 & $.013 *$ & 0.329 & $.019 *$ & -0.044 & .760 & 0.021 & .941 \\
\hline Lymphocytes & -0.194 & .119 & 0.139 & .346 & -0.084 & .496 & -0.037 & .808 & -0.078 & .571 & 0.113 & .413 \\
\hline Neutrophils & 0.128 & .586 & 0.273 & .052 & 0.088 & .676 & 0.085 & .769 & -0.005 & .974 & 0.400 & $.003 *$ \\
\hline gender & 0.258 & $.016 *$ & 0.256 & .077 & 0.059 & .625 & 0.083 & .596 & 0.162 & .271 & 0.165 & .208 \\
\hline BMI & -0.160 & .332 & -0.298 & .095 & -0.357 & $.023 *$ & 0.406 & $.041 *$ & -0.644 & $.003 *$ & -0.315 & .102 \\
\hline Diastolic BP & -0.072 & .496 & -0.165 & .245 & -0.005 & .963 & -0.072 & .609 & -0.081 & .560 & 0.109 & .397 \\
\hline Systolic BP & 0.001 & .990 & -0.168 & .237 & -0.123 & .266 & -0.088 & .530 & -0.297 & $.034 *$ & -0.068 & .589 \\
\hline $\mathrm{R} 2$ & \multicolumn{2}{|c|}{0.212} & \multicolumn{2}{|c|}{0.259} & \multicolumn{2}{|c|}{0.452} & \multicolumn{2}{|c|}{0.446} & \multicolumn{2}{|c|}{0.308} & \multicolumn{2}{|c|}{0.338} \\
\hline
\end{tabular}

AHA indicates American Heart Association; BP, blood pressure; FH, family history of cerebrovascular symptomatology; HDL, high density lipoprotein; hsCRP, high sensitivity C-reactive protein; HbA1c, glycated hemoglobin; LDL, low density lipoprotein; WC, waist circumference. *Significant P values for non-adjusted and adjusted Spearman correlation with plasma resistin levels.

ischemic stroke [Park 2013; Naghavi 2003; Perovic 2017; Efstathiou 2007; Prugger 2012; Prugger 2013]. Resistin is found in human atherosclerotic lesions, and some studies suggest that elevated resistin levels may also serve as a potential marker of subclinical carotid atherosclerosis. [Park 2013; Ntaios 2013; Yang 2018].

Quite recently, elevated resistin serum levels were also related to overall cerebrovascular symptomatology in patients with moderate to high grades of CAS [Gasbarrino 2016]. However, no association between serum resistin and plaque instability was detected and its association with carotid artery stenosis-induced ischemic stroke events remains elusive.

The objectives of this study were to evaluate the association between both serum and intraplaque resistin levels with ischemic stroke events and histological features of carotid plaque instability in patients with moderate to high grades of CAS who were scheduled for elective carotid endarterectomy. We also evaluated the association between serum and intraplaque resistin levels.

Taking into the account the reported association between serum resistin levels and ischemic stroke and a documented presence of resistin expression in human atherosclerotic lesions, we sought to study the positive association between circulatory and carotid plaque resistin levels and their direct association with plaque instability and ischemic stroke risk.

\section{PATIENTS AND METHODS}

A total of 78 consecutive neurologically symptomatic (ischemic stroke, $n=40)$ and asymptomatic $(n=38)$ patients with moderate- to high-grade carotid artery stenosis (CAS) scheduled for CEA between February 2013 and July 2014 at the Department of Surgery, University Hospital Dubrava (UHD), Zagreb, Croatia, were included in this study. CAS was diagnosed and classified by the LOGIQ* E9 Doppler ultrasound machine according to consensus criteria [von Reutern 2012; Grant 2003]. In the case of inconclusive findings an additional imaging technique was also applied (computed tomography or magnetic resonance angiography). Patients were defined as symptomatic if they had recently $(<6$ months prior to CEA) developed ischemic stroke ipsilateral to the internal carotid artery stenosis, whereas the patients who experienced cerebrovascular symptoms $\geq 6$ months prior to CEA or never at all were classified as asymptomatic. To avoid the confounding effect of other clinical entities on serum resistin levels, obese patients $(\mathrm{BMI}>35)$, patients with acute or chronic infectious diseases, autoimmune diseases, malignant diseases, renal failure, and patients with serious hepatic diseases were excluded from the study. All patients with possible non-carotid sources of ischemic symptoms were excluded as well. Written informed consent was obtained 
Table 5. Prevalence of Histological Plaque Features According to Ischemic Stroke and Plaque Instability

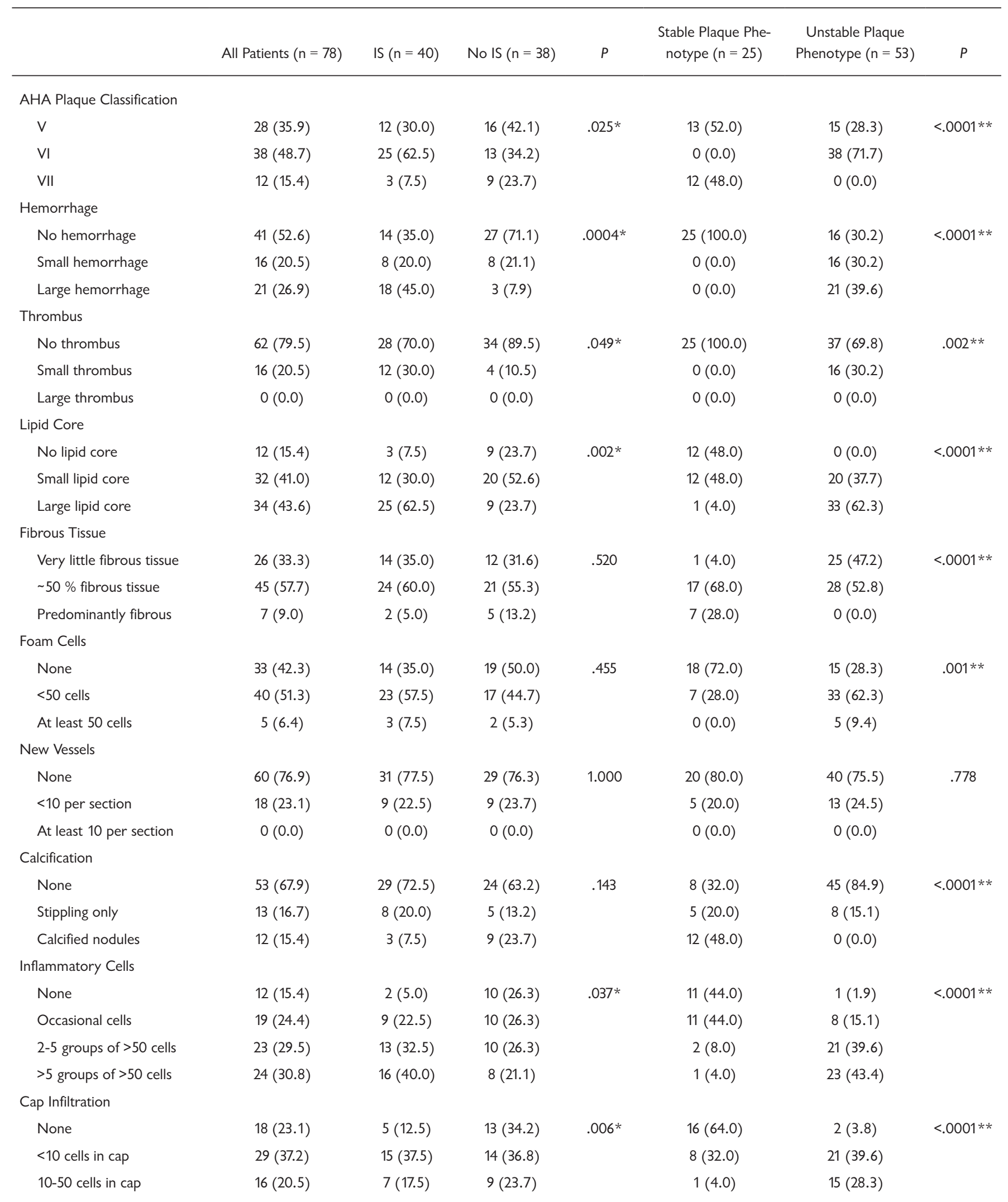


Table 5. Prevalence of Histological Plaque Features According to Ischemic Stroke and Plaque Instability

\begin{tabular}{|c|c|c|c|c|c|c|c|}
\hline \multicolumn{8}{|l|}{ Rupture } \\
\hline Intact cap & $39(50.0)$ & $16(40.0)$ & $23(60.5)$ & $.001 *$ & $23(92.0)$ & $16(30.2)$ & $<.0001 * *$ \\
\hline Definitely ruptured & $14(17.9)$ & $12(30.0)$ & $2(5.3)$ & & $0(0.0)$ & $14(26.4)$ & \\
\hline \multicolumn{8}{|l|}{ Overall Instability } \\
\hline Definitely stable & $10(12.8)$ & $2(5.0)$ & $8(21.1)$ & $.0002 *$ & $10(40.0)$ & $0(0.0)$ & $<.0001 * *$ \\
\hline Probably stable & $15(19.2)$ & $3(7.5)$ & $12(31.6)$ & & $15(60.0)$ & $0(0.0)$ & \\
\hline High (>25\% of + cells) & $31(39.7)$ & $20(50.0)$ & $11(28.9)$ & $.068 *$ & $1(4.0)$ & $30(56.6)$ & $<.0001 * *$ \\
\hline Low $(0 \%-25 \%$ of + cells $)$ & $47(60.3)$ & $20(50.0)$ & $27(71.1)$ & & $24(96)$ & $23(43.4)$ & \\
\hline
\end{tabular}

AHA indicates American Heart Association; IS, ischemic stroke; *P (Fisher exact or Pearson chi-square test as appropriate) value indicates significance between patients with ischemic stroke and non-symptomatic patients; **P (Fisher exact test) value indicates significance between patients with stable and patients with unstable plaques.

from all subjects and the study was approved by the Ethics Committee of UHD.

\section{Clinical and Biochemical Assessment}

Patient demographic and clinical data (past medical history, cardiovascular and cerebrovascular symptomatology, presence of cardiovascular risk factors, medication usage, lifestyle habits, and family history of cerebrovascular diseases) were recorded (patient interview, questionnaire, and medical records) from all patients upon entering the study. Blood pressure (BP) was measured with mercurial sphygmomanometer (Riester, Jungingen, Germany) and calculated as the average of three measurements taken under standardized conditions in a supine position. Height, weight, and waist circumference (WC) were measured by the same observer. The body mass index (BMI) was calculated according to Quetelet's formula as the ratio of body weight to body height squared $\left(\mathrm{kg} / \mathrm{m}^{2}\right)$. Blood samples for laboratory assays were obtained prior to CEA at approximately $8 \mathrm{am}$, following overnight fasting. The blood samples were centrifuged (3000 g for $10 \mathrm{~min}$ ) to obtain serum, and rapidly stored at $-80 \mathrm{oC}$ until analysis. Serum lipid profile (triglycerides, total cholesterol, low-density lipoprotein cholesterol [LDL], and high-density lipoprotein cholesterol [HDL]), complete blood cell count, fibrinogen, high-sensitivity C-reactive protein (hs$\mathrm{CRP}$ ) and glycated hemoglobin (HbA1C) were measured in the central biochemistry laboratory of the UHD using standardized methods and protocols. The serum resistin concentration was measured using enzyme-linked immunosorbent assay (ELISA; eBioscience, Vienna, Austria). All samples were run in duplicates with quality controls, and inter- and intra-assay coefficients of variation were $<10 \%$.

\section{Tissue Sampling, Histological Characterization of Carotid Atherosclerotic Plaques}

Atherosclerotic carotid plaques were retrieved from patients during carotid endarterectomy. Immediately after surgical resection, plaques were dissected into transverse segments approximately $3-4 \mathrm{~mm}$ in thickness, depending upon the plaque size, fixed in 10\% formalin, decalcified (Osteosoft, Merck) and then processed for paraffin embedment. Plaque morphology and stability was assessed by standard histological examination of 4- $\mu \mathrm{m}$ thick hematoxylin and eosin-stained sections (from the area of maximum stenosis or with the largest plaque burden) and characterized according to histological classification of the American Heart Association (AHA) reported by Stary et al, and 3- to 4-grade semi-quantitative scale described by Lovett et al (presence or absence of: hemorrhage, thrombosis, foam cells, fibrous tissue, new vessel formation, cap rupture, calcification, lipid core size, overall intraplaque inflammatory cell infiltration [the number of macrophages and lymphocytes present] and inflammatory infiltration of the fibrous cap) [Perovic 2017; Prugger 2012; Prugger 2013]. All histological analyses were performed by two experienced vascular pathologists blinded to the study outcome.

\section{Immunobistochemistry}

Paraformaldehyde-fixed sections (4- $\mu \mathrm{m})$ of asymptomatic and symptomatic carotid plaques were stained with the 
Table 6. Univariate and Multivariate Logistic Regression Analyses for the Association of Plasma Resistin Levels with Ischemic Stroke (symptomatic $[n=40]$ versus asymptomatic patients [ $n=38]$ ), Unstable Plaque Phenotype (stable $[n=25]$ versus unstable $[n=$ 53]), Type 2 Diabetic Patients with (ischemic stroke [ $n=23]$ versus No Ischemic Stroke without [ $n=22]$ ), Patients without Type 2 Diabetes (ischemic stroke [ $n=17$ ] versus no ischemic stroke $[n=16]$ ), and Plaque Resistin Levels (low [ $n=47]$ versus high [ $n=31]$ )

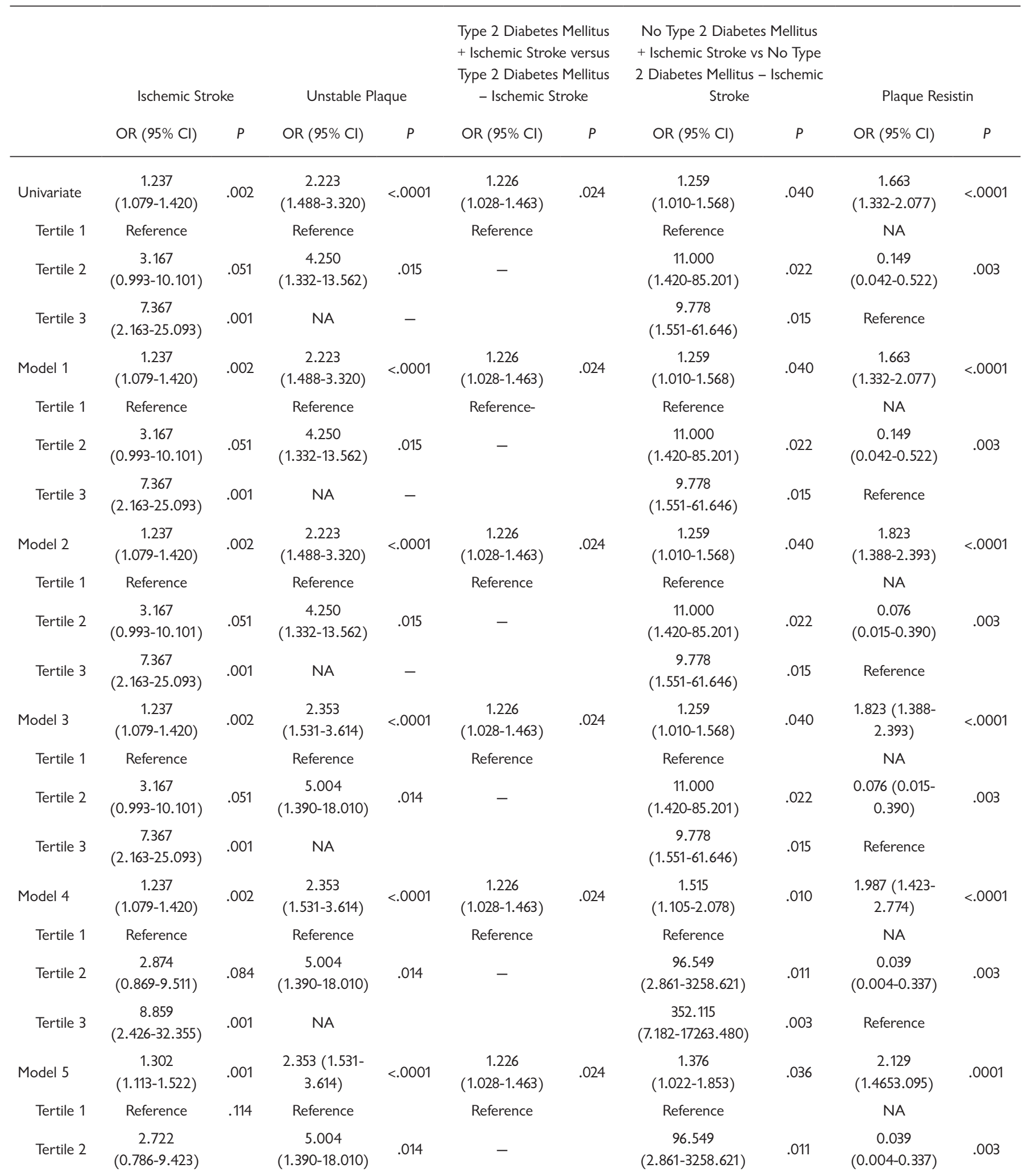


Table 6. Univariate and Multivariate Logistic Regression Analyses for the Association of Plasma Resistin Levels with Ischemic Stroke (symptomatic $[n=40]$ versus asymptomatic patients $[n=38]$ ), Unstable Plaque Phenotype (stable $[n=25]$ versus unstable $[n=$ 53]), Type 2 Diabetic Patients with (ischemic stroke [ $n=23]$ versus No Ischemic Stroke without [ $n=22]$ ), Patients without Type 2 Diabetes (ischemic stroke $[n=17]$ versus no ischemic stroke $[n=16]$ ), and Plaque Resistin Levels (low [ $n=47]$ versus high [ $n=31]$ )

\begin{tabular}{|c|c|c|c|c|c|c|c|c|c|c|}
\hline & \multicolumn{2}{|c|}{ Ischemic Stroke } & \multicolumn{2}{|c|}{ Unstable Plaque } & \multicolumn{2}{|c|}{$\begin{array}{l}\text { Type } 2 \text { Diabetes Mellitus } \\
\text { + Ischemic Stroke versus } \\
\text { Type } 2 \text { Diabetes Mellitus } \\
\text { - Ischemic Stroke }\end{array}$} & \multicolumn{2}{|c|}{$\begin{array}{c}\text { No Type } 2 \text { Diabetes Mellitus } \\
+ \text { Ischemic Stroke vs No Type } \\
2 \text { Diabetes Mellitus - Ischemic } \\
\text { Stroke }\end{array}$} & \multicolumn{2}{|c|}{ Plaque Resistin } \\
\hline & OR $(95 \% \mathrm{Cl})$ & $P$ & OR $(95 \% \mathrm{Cl})$ & $P$ & OR $(95 \% \mathrm{Cl})$ & $P$ & OR $(95 \% \mathrm{Cl})$ & $P$ & OR $(95 \% \mathrm{Cl})$ & $P$ \\
\hline Model 6 & $\begin{array}{c}1.302 \\
(1.113-1.522)\end{array}$ & .001 & $\begin{array}{c}2.353 \\
(1.531-3.614)\end{array}$ & $<.0001$ & $\begin{array}{c}1.226 \\
(1.028-1.463)\end{array}$ & .024 & $\begin{array}{c}2.593 \\
(1.132-5.935)\end{array}$ & .024 & $\begin{array}{c}2.129 \\
(1.4653 .095)\end{array}$ & .0001 \\
\hline Tertile 1 & Reference & & Reference & & Reference & & Reference & & NA & \\
\hline Tertile 2 & $\begin{array}{c}2.722 \\
(0.786-9.423)\end{array}$ & .114 & $\begin{array}{c}5.004 \\
(1.390-18.010)\end{array}$ & .014 & - & & NA & & $\begin{array}{c}0.039 \\
(0.004-0.337)\end{array}$ & .003 \\
\hline
\end{tabular}

Model 1 indicates partially adjusted for age, sex, and BMI; Model 2, Model 1 + type 2 diabetes and hsCRP; Model 3, Model $2+$ waist circumference; Model 4, Model 3 + smoking, hyperlipidemia, total cholesterol, triglyceride, HDL, LDL, leukocytes, neutrophils, lymphocytes, systolic and diastolic blood pressure; Model 5 , Model 4 + family history of cerebrovascular symptomatology, peripheral artery disease, coronary artery disease, arterial hypertension, pulmonary hypertension, aortic stenosis and carotid artery stenosis (79\%-99\% versus 60\%-79\%); Model 6 (fully-adjusted model), Model $5+$ medications (statins, insulin, ACE inhibitors, beta blockers, calcium channel blockers and antihyperglycemic) usage. NA, not applicable.

use of primary antibody for human resistin (Resistin/RETN antibody, eBioscience) followed by EnVision antibody-HRP (horseradish peroxidase)-enzyme conjugate complex (DAKO; Hamburg, Germany). Diaminobenzidine (DAB) was used as the chromogen and the complete reaction was performed in automated autostainer (DAKO; Hamburg, Germany) according to the manufacturer's instructions. The sections were counterstained with hematoxylin. Omission of the primary antibody served as a negative control whereas the positive control samples were represented by fatty tissue around the kidney with xanthogranulomatous inflammation. The expression of resistin was evaluated on a semi-quantitative scale by two experienced vascular pathologists blinded to the clinical and histology data. The scoring was arbitrary, as follows: 0 without staining, 1 - rare, focal staining $(<25 \%$ positive cells), 3 - high staining (>25\% positive cells).

\section{Statistical Analysis}

Results are expressed as counts (n) and percentages (\%) for categorical variables and as the mean \pm standard deviation (SD) for normally distributed continuous variables or as the median and interquartile range (IQR) for continuous variables with nonparametric distribution. Data distribution was analyzed by Shapiro-Wilk test. Differences between groups were evaluated by independent samples $t$ test and/or analysis of variance (ANOVA) for normally distributed continuous variables and by Mann-Whitney or Kruskal-Wallis test for continuous variables with nonparametric distribution. Qualitative and categorical variables were compared by the Pearson chi-square or Fisher exact test as appropriate. The correlation between serum resistin levels and the other continuous variables was performed with bivariate (Pearson or Spearman-rank correlation coefficient) and partial (adjusted by age, sex, and BMI) regression analysis. Associations between serum resistin levels as dependent variable and multiple independent variables were tested using stepwise linear regression analysis (F-value probability for inclusion of 0.05 and 0.01 for removal), while those with categorical dependent variables were tested using univariate and multivariate stepwise logistic regression analysis. Multivariate logistic regression analyses were presented as 6 adjusted models: Model 1 - partially adjusted for age, sex, and BMI; Model 2 - Model $1+$ T2DM and hsCRP; Model 3 Model 2 + waist circumference; Model 4 - Model 3 + smoking, hyperlipidemia, total cholesterol, triglyceride, HDL, LDL, leukocytes, neutrophils, lymphocytes, systolic and diastolic blood pressure; Model 5 - Model 4 + family history of cerebrovascular symptomatology, peripheral artery disease, coronary artery disease, arterial hypertension, pulmonary hypertension, aortic stenosis and carotid artery stenosis (79\%-99\% versus 60\%-79\%) and Model 6 (fully-adjusted model) - Model $5+$ medications (statins, insulin, ACE inhibitors, beta blockers, calcium channel blockers and antihyperglycemic) usage. Odds ratios (OR) are presented with $95 \%$ confidence intervals (CI). For statistical analysis "definitely stable" and "probably stable" plaque groups classified by the semi-quantitative scale of plaque instability were combined together and designated 
Table 7. Univariate and Multivariate Logistic Regression Analyses for the Association of Plasma Resistin Levels with AHA Plaque Classification and Plaque Histological Features (hemorrhage, thrombus, and lipid core presence)




Table 7. Univariate and Multivariate Logistic Regression Analyses for the Association of Plasma Resistin Levels with AHA Plaque Classification and Plaque Histological Features (hemorrhage, thrombus, and lipid core presence)



Model 1 indicates partially adjusted for age, sex, and BMI; Model 2, Model 1 + type 2 diabetes and hsCRP; Model 3, Model 2 + waist circumference; Model 4, Model 3 + smoking, hyperlipidemia, total cholesterol, triglyceride, HDL, LDL, leukocytes, neutrophils, lymphocytes, systolic and diastolic blood pressure; Model 5 , Model 4 + family history of cerebrovascular symptomatology, peripheral artery disease, coronary artery disease, arterial hypertension, pulmonary hypertension, aortic stenosis and carotid artery stenosis (79\%-99\% versus 60\%-79\%); Model 6 (fully-adjusted model), Model $5+$ medications (statins, insulin, ACE inhibitors, beta blockers, calcium channel blockers and antihyperglycemic) usage. NA, not applicable. [TBL]Table 8. Univariate and Multivariate Logistic Regression Analyses for the Association of Plasma Resistin Levels with Plaque Histological Features (fibrous tissue, foam cells, calcification, inflammation, cap infiltration and rupture)

as "stable," while the "probably unstable" and "definitely unstable" plaque groups combined together were designated as "unstable" and as such used in the statistical analyses. For the same reasons immunohistochemical scoring of the resistin plaque expression was divided in to two groups: low (0-25\% of + cells) and high staining ( $>25 \%$ of + cells). All statistical analyses were performed in SPSS, Version 22 (IBM, Armonk, New York, USA). A two-sided probability of less than .05 was considered statistically significant.

\section{RESULTS}

A total of 78 patients included in this study were stratified in four groups: ischemic stroke and asymptomatic patient group and stable and unstable plaque phenotype group (Table 1).

In the ischemic stroke group, a significantly higher incidence of patients with unstable plaque phenotype, higher serum resistin level, and family history of cerebrovascular symptoms were found (Table 1). Systolic BP was also higher in this group, but the difference did not reach statistical significance (Table 1). In contrast, subjects with unstable carotid plaque were more likely to be men than subjects with stable plaque phenotype (Table 1). An elevated serum resistin level was also found in the group with unstable plaque phenotype. Furthermore, the number of patients with family history of cerebrovascular symptomatology and of patients treated with calcium channel blockers was also higher in this group but the difference did not reach statistical significance (Table 1). No other differences in terms of patient demographic, medication, or clinical, biochemical, and hematological parameters were noted between the groups (Table 1).
When patients were analyzed according to sex and T2DM status, male subjects had significantly higher circulatory resistin levels $(8.509$ [4.915-11.105] ng/mL versus 4.778 [4.275$7.306] \mathrm{ng} / \mathrm{mL}, P=.01$ ), while there was no diference in serum resistin values between T2DM and non-diabetic patients (6.787 [4.556-10.167] $\mathrm{ng} / \mathrm{mL}$ versus 5.209 [4.317-10.220] ng/ $\mathrm{mL}, P=.457)$. However, among patients with T2DM, resistin levels were significantly elevated in patients with ischemic stroke versus asymptomatic subjects $(8.625[6.245-11.105] \mathrm{ng} /$ $\mathrm{mL}$ versus 5.520 [3.876-8.803] $\mathrm{ng} / \mathrm{mL}, P=.012)$. Serum resistin levels were also significantly elevated in ischemic stroke versus asymptomatic patients without T2DM (8.952 [5.161$11.242] \mathrm{ng} / \mathrm{mL}$ versus 4.364 [3.449-7.505] ng/mL, $P=.005)$.

Also, patients with positive family history of cerebrovascular symptomatology exhibited higher although marginally insignificant serum resistin levels when compared to subjects without family history of cerebrovascular symptomatology $(7.443[4.673-10.249] \mathrm{ng} / \mathrm{mL}$ versus 5.450 [3.608-8.943] $\mathrm{ng} /$ $\mathrm{mL}, P=.075)$. In addition, subjects with T2DM were more frequently hypertensive $(P=.003)$, had significantly higher systolic BP (140.00 [130.00-140.00] versus 130.00 [120.00$140.00], P=.013)$ and diastolic BP (90.00 [80.00-92.50] versus 80.00 [80.00-87.50, $P=.05$ ), and, as expected, significantly higher serum HbA1c $(P<.0001)$ levels, when compared to people with normal glycaemia.

\section{Correlation and Linear Regression Analysis}

In the non-adjusted Spearman correlation analysis of the whole group, serum resistin was positively correlated with leukocytes and neutrophils and marginally insignificantly with waist circumference, while in the subgroup of patients 
Table 8. Univariate and Multivariate Logistic Regression Analyses for the Association of Plasma Resistin Levels with Plaque Histological Features (fibrous tissue, foam cells, calcification, inflammation, cap infiltration and rupture)

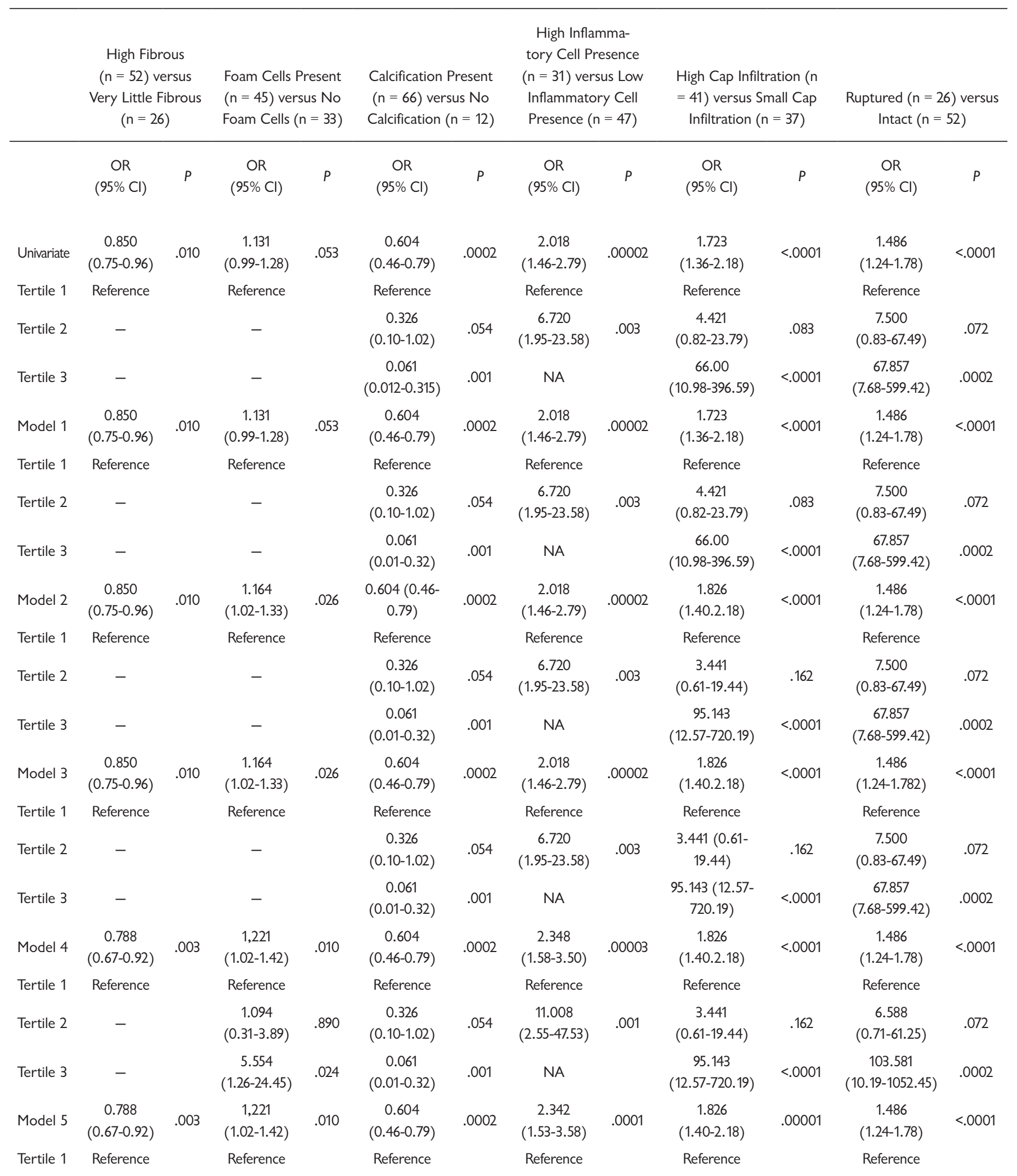




\begin{tabular}{|c|c|c|c|c|c|c|c|c|c|c|c|c|}
\hline Tertile 3 & - & & $\begin{array}{c}5.554 \\
(1.26-24.45)\end{array}$ & .024 & $\begin{array}{c}0.061 \\
(0.01-0.32)\end{array}$ & .001 & NA & & $\begin{array}{c}95.143 \\
(12.57-720.19)\end{array}$ & $<.0001$ & $\begin{array}{c}103.581 \\
(10.19-1052.45)\end{array}$ & .0002 \\
\hline Model 6 & $\begin{array}{c}0.788 \\
(0.67-0.92)\end{array}$ & .003 & $\begin{array}{c}1,221 \\
(1.02-1.42)\end{array}$ & .010 & $\begin{array}{c}0.604 \\
(0.46-0.79)\end{array}$ & .0002 & $\begin{array}{c}2.342 \\
(1.53-3.58)\end{array}$ & .0001 & $\begin{array}{c}2.214 \\
(1.53-3.20)\end{array}$ & $<.0001$ & $\begin{array}{c}1.561 \\
(1.26-1.93)\end{array}$ & $<.0001$ \\
\hline Tertile 3 & & & $\begin{array}{c}5.554 \\
(1.26-24.45)\end{array}$ & .024 & $\begin{array}{c}0.061 \\
(0.01-0.32)\end{array}$ & .001 & NA & & $\begin{array}{c}315.996 \\
(24.97-3998.76)\end{array}$ & $<.0001$ & $\begin{array}{c}88.525 \\
(8.75-895.91)\end{array}$ & .0002 \\
\hline
\end{tabular}

Model 1 indicates partially adjusted for age, sex, and BMI; Model 2, Model 1 + type 2 diabetes and hsCRP; Model 3, Model 2 + waist circumference; Model 4, Model 3 + smoking, hyperlipidemia, total cholesterol, triglyceride, HDL, LDL, leukocytes, neutrophils, lymphocytes, systolic and diastolic blood pressure; Model 5 , Model 4 + family history of cerebrovascular symptomatology, peripheral artery disease, coronary artery disease, arterial hypertension, pulmonary hypertension, aortic stenosis and carotid artery stenosis (79\%-99\% versus 60\%-79\%); Model 6 (fully-adjusted model) - Model $5+$ medications (statins, insulin, ACE inhibitors, beta blockers, calcium channel blockers and antihyperglycemic) usage. NA, not applicable.

with ischemic stroke it was positively correlated with waist circumference, triglycerides, leukocytes, and neutrophils (Table 2). Also, in the non-adjusted Spearman correlation analysis of the whole patient group with unstable plaque, phenotype serum resistin was positively correlated with waist circumference, triglyceride, leukocytes and neutrophils (Table 2). In contrast, in the Spearman partial correlation analysis of the whole patient group, adjusted by age, sex, and BMI, serum resistin was positively correlated with waist circumference, leukocytes, and neutrophils, while in the subgroup of patients with ischemic stroke it was correlated only with waist circumference, and marginally insignificantly with leukocytes (Table 2). Furthermore, in the group of patients with unstable plaque, phenotype serum resistin was positively correlated with waist circumference, leukocytes, triglycerides, and neutrophils (Table 2).

Circulating resistin levels were also positively correlated with waist circumference, leukocytes, neutrophils, and triglycerides in the patient group with AHA plaque classification type VI and positive family history of cerebrovascular symptomatology (non-adjusted and adjusted Spearman correlation coefficients, correspondingly); only with waist circumference in the patient group with T2DM (adjusted Spearman correlation coefficients); and with lymphocytes and HDL-cholesterol (significant non-adjusted and marginally insignificant adjusted Spearman correlation coefficients) in the patient group with family history of cerebrovascular symptomatology (Table 3). Non-adjusted Spearman correlation also showed a significant correlation between male sex and serum resistin levels in the overall $(\beta=0.0293, P=.009)$ and ischemic stroke ( $\beta=0.380, P=.015)$ patient group (data not shown).

In addition, multiple stepwise linear regression analysis revealed waist circumference and leukocytes as independent positive predictors of serum resistin levels in the whole patient group and in subgroups with ischemic stroke (leukocytes marginally insignificant), unstable plaque phenotype, and AHA plaque type VI (Table 4). Waist circumference was also an independent predictor of serum resistin levels in the patient subgroup with T2DM and a family history of cerebrovascular symptomatology (Table 4).

In contrast, neutrophils and sex were significant positive predictors of serum resistin levels only for the patient subgroup with a family history of cerebrovascular symptomatology and in the whole patient group, respectively.

Furthermore, multiple stepwise linear regression analysis was performed according to sex (data not shown), and leukocytes were only a significant predictor of serum resistin levels in the male sex subgroup $(\beta=0.369, P=.010)$. Also, BMI and systolic BP were the only negatively related predictors of serum resistin levels with significant negative effect of BMI in patient groups with unstable plaque phenotype, AHA plaque type VI and T2DM and systolic BP in patient subgroup with T2DM (Table 4).

\section{Prevalence of Plaque Histological Features}

Surgical specimens obtained from the CEA were all representative of advanced (Type V-VII) atherosclerotic lesions with statistically significant prevalence of AHA plaque classification type VI among patients with ischemic stroke and unstable plaque phenotype (Table 2).

Furthermore, except for neovascularization, all other histological features of obtained carotid atherosclerotic plaques showed significantly different prevalence in patients with stable versus unstable plaque phenotype (Table 5). In contrast, in addition to the level of neovascularization, patient subgroups stratified according to ischemic stroke also showed no significant difference in the proportion of: fibrous tissue formation, foam cells presence, and calcification grade (Table 5).

\section{Resistin Plaque Immunostaining}

Resistin intraplaque immunostaining was significantly higher in unstable compared to stable carotid plaques and marginally insignificant in ischemic stroke versus the asymptomatic patient group (Table 5). A significant difference in resistin immunostaining was also observed according to AHA plaque classification (Fisher exact test, $P<.0001)$ with high $(>25 \%$ of 

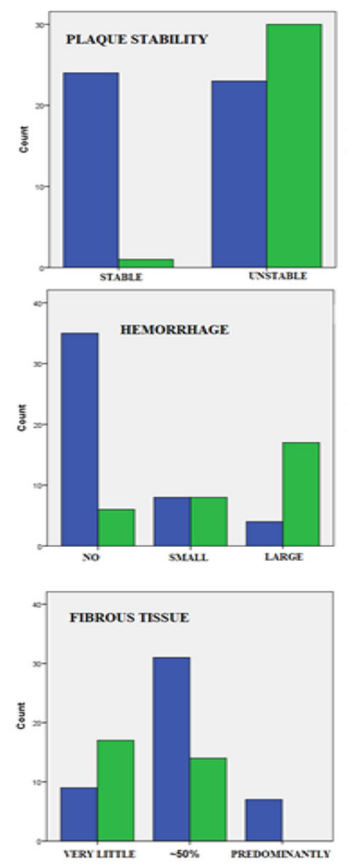
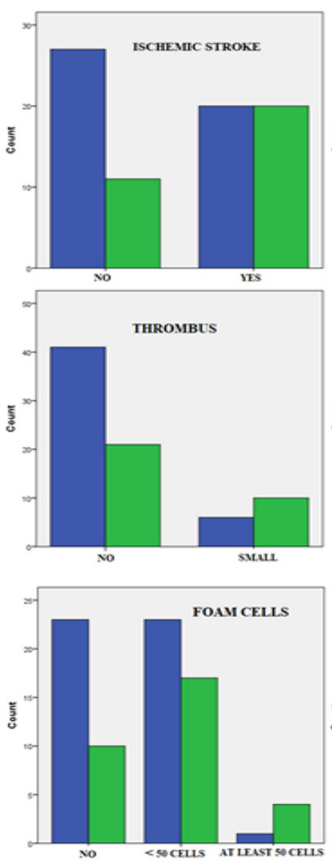
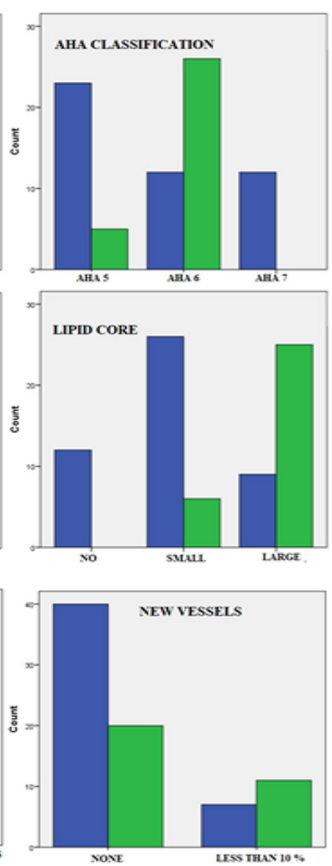
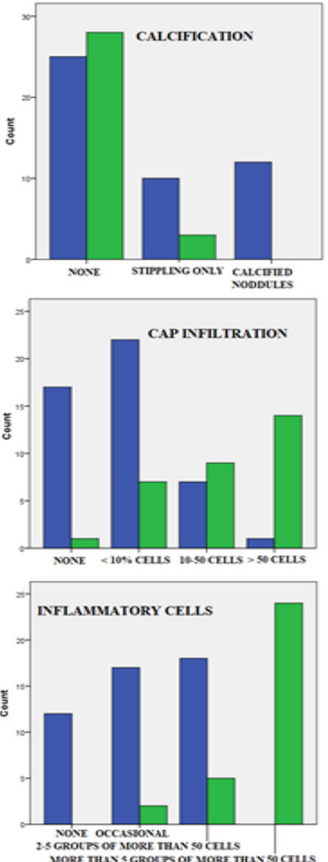
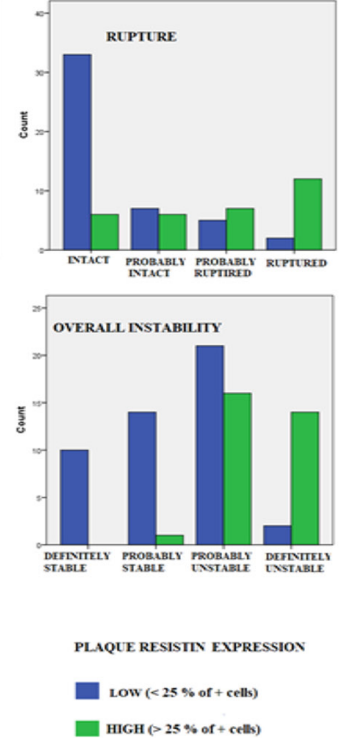

HIGH (>25\% or + cethis)

Figure 1. Distribution of low (<25\% of + cells) and high ( $>25 \%$ of + cells) grade of plaque resistin expression according to AHA (American Heart Association) plaque type classification and semi-quantitative plaque histological features.

+ cells) intensity of immunostaining most frequently observed in AHA plaque classification type VI (Figure 1). Furthermore, according to the semi-quantitative scale of histological plaque features, significant difference (Fisher exact or Pearson chisquare test as appropriate) in resistin immunostaining was also observed regarding the presence/absence of intraplaque hemorrhage $(P<.0001)$, thrombus (Pearson chi-square, $\chi^{2}=26.388$, $\mathrm{df}=2, P=.048)$, lipid core $(P<.0001)$, fibrous tissue formation $(P=.001)$, calcification $(P=.001)$, inflammatory cells presence $(P<.0001)$, fibrous cap infiltration $(P<.0001)$, and rupture $(P$ $<.0001)$; and marginally insignificant difference regarding the new vessel formation (Pearson chi-square, $\chi^{2}=4.461, \mathrm{df}=1, P$ $=.054)$ and foam cell presence $(P=.086)$ (Figure 1$)$.

Low levels of resistin expression were observed in carotid plaques with calcified nodules (no expression) and predominantly fibrous plaques $(0 \%-25 \%$ of + cells $)$, while the highest $(>25 \%$ of + cells) resistin expression was observed in plaques with large lipid core (Figure 2) and higher presence of inflammatory cell (Figure 1).

In addition, univariate and multivariate models of stepwise logistic regression analysis revealed significant positive association of elevated serum resistin levels with intraplaque resistin expression, showing more than $60 \%$ increase in odds ratio for carotid plaques with higher level (>25\% of + cells) of resistin expression (Table 6).

\section{Association of Serum Resistin Levels with Carotid Plaque Instability and Ischemic Stroke Symptomatology}

Serum resistin levels were significantly associated with plaque instability, with more than 2 times higher serum resistin levels in the patient group with unstable versus stable plaques, both in the univariate and multivariate models of stepwise logistic regression analysis (Table 6). There was also a significant 4- to 5-fold increase in the odds ratio for plaque instability when the second tertile of resistin serum levels was compared with the lowest (Table 6).

Univariate model of stepwise logistic regression analysis also showed significant elevation of serum resistin levels (more than 20\% increase) in ischemic stroke versus asymptomatic patients (Table 6). Furthermore, there was significant increase in the odds ratio for ischemic stroke when the highest tertile of serum resistin levels were compared with the lowest. In addition, elevated resistin levels were also significantly associated with ischemic stroke both in subjects with and without T2DM (Table 6). These associations remained significant even after additional adjustments for hematological, metabolic, and cardiovascular risk factors as well as

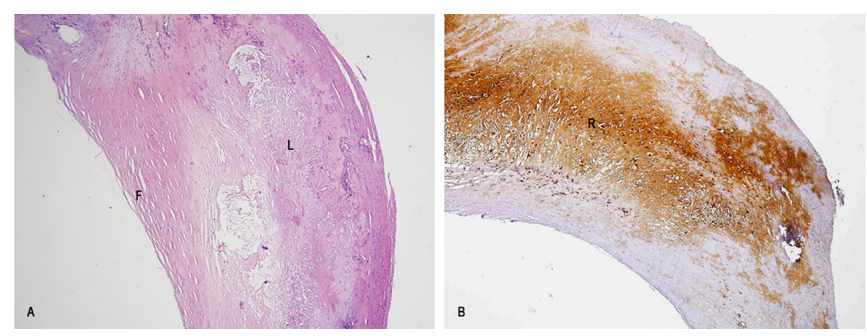

Figure 2. A, Mixed fibrolipid plaque with lipid core (L) and fibrous cap $(\mathrm{F})$; $\mathrm{B}$, High resistin (R) immunoreactivity in plaque lipid core. 
for present comorbidities and when medications used were applied (Models 2-6) (Table 6).

No association was found between serum resistin levels and degree of carotid artery stenosis $(80 \%-99 \%$ versus $60 \%-$ $79 \%$; data not shown).

\section{Association of Serum Resistin Levels with Histological Fea- tures of Carotid Atherosclerotic Plaque}

Significant association was found between serum resistin levels and AHA plaque classification types in their overall $(P<.0001)$ and mutual comparison (VI versus V: $P<.0001$; VI versus VII: $P<.0001$; V versus VII: $P=.001)$ with highest serum resistin levels found in patients with AHA plaque classification type VI and lowest in AHA plaque classification type VII (median and IQR range, AHA type V: 4.702 [4.3785.878]; AHA type VI: 10.220 [8.352-13.825]; AHA type VII: 2.780 [2.218-3.820]). This was confirmed with univariate and multivariate logistic regression analysis showing significant association (2.8x higher increase in odds ratio) of elevated serum resistin levels with AHA plaque type VI versus type $\mathrm{V}$ and significantly lower association with AHA plaque type VII versus type $\mathrm{V}$ and VI combined together (Table 7).

Elevated serum resistin levels also increased ( $>2$-fold) the odds ratio of having plaques with lipid core presence and high levels of inflammatory cells (Tables 7 and 8). They were also significantly associated with increased intraplaque presence of: inflammatory cap infiltration, large hemorrhage, thrombus, foam cell and cap rupture. In contrast to that, lower levels of serum resistin were associated with increased odds ratio of having plaques with presence of intraplaque calcification and higher proportion of fibrosis. These associations remained significant in all models of multivariate logistic regression analysis (Tables 7 and 8).

No association was observed between serum resistin levels and new vessel formation in either univariate or multivariate logistic regression analysis (data not shown).

\section{DISCUSSION}

The major findings of the present study are: (1) the higher levels of serum resistin levels were associated with increased odds of ischemic stroke events and carotid plaque instability; (2) elevated serum resistin levels were also associated with AHA plaque classification type VI and all semi-quantitative histological plaque features responsible for higher level of carotid plaque vulnerability; (3) higher odds of intraplaque resistin expression were positively associated with elevated serum resistin levels and; (4) higher levels of intraplaque resistin expression were associated with increased odds of plaque instability and all histological features related to a higher level of plaque vulnerability. These associations remained significant even after additional adjustments for hematological, metabolic, and cardiovascular risk factors as well as for present comorbidities and medications used. Likewise, decreased serum and intraplaque resistin levels were associated with increased odds of AHA plaque classification type VII and semi-quantitative histological features associated with the decreased odds of plaque vulnerability such as the higher degree of intraplaque fibrosis and calcification. In contrast, intraplaque resistin expression was only marginally associated with recent ischemic stroke symptomatology and no association of serum or intraplaque resistin levels was found with the degree of carotid artery stenosis.

To the best of our knowledge, this is the first study showing the association of elevated circulatory and plaque resistin levels with ischemic stroke events and carotid plaque vulnerability among CAS patients subjected to CAE.

Our data are in agreement with previous studies reporting the significant association of elevated serum resistin levels with an increased incidence of acute ischemic stroke [Naghavi 2003; Perovic 2017; Efstathiou 2007; Prugger 2012; Prugger 2013].

As already stated, significant and independent association between increased resistin serum levels and symptomatology in patients with moderate to high grade of CAS scheduled for CEA was previously reported by Gasbarrino et al [Gasbarrino 2016]. However, in their study elevated serum resistin levels were associated with overall cerebrovascular symptomatology in patients with T2DM while the association with individual types of cerebrovascular events (ie, highest levels of serum resistin detected in patients who had a stroke and lowest in patients with history of amaurosis fugax) were detected primarily in subjects without T2DM [Gasbarrino 2016]. In contrast to that in our data, elevated resistin levels were detected both in ischemic stroke patients with and without T2DM. They also reported no association of serum resistin levels with histological features of plaque instability [Gasbarrino 2016]. This discrepancy in results may be explained by the fact that the individual subjects included in their study experienced ( $<6$ months prior to CEA) various aspects of cerebrovascular symptomatology (stroke, transient ischemic attack, or amaurosis fugax), while the subjects with neurovascular symptomatology included in our study experienced only ischemic stroke events. In contrast to results reported by Gasbarrino et al, our study showed significant association of higher serum resistin levels in vulnerable AHA plaque type VI (the most complicated lesion characterized by the presence of hematoma, hemorrhage, or thrombus) in comparison to AHA plaque type $\mathrm{V}$ (fibroatheroma with a large lipid core) and VII (predominantly fibrotic/calcific) [Gasbarrino 2016]. The same was true for the association of higher serum resistin in AHA plaque type V versus type VII. Since clinical outcomes are most often associated with AHA plaque type $\mathrm{V}$ and $\mathrm{VI}$, these results indicate that serum resistin levels may act as potential markers for plaque instability.

Resistin is known to be expressed in human atherosclerotic plaques and its proatherogenic actions are most probably associated with increased macrophage infiltration, upregulation of proinflammatory cytokines and adhesion molecules in human endothelial cells, increased smooth muscle cell proliferation and the formation of foam cells [Park 2013; Kunnari 2006; Xu 2006; Cho 2011; Verma 2003; Calabro 2004; Jung 2006]. Furthermore, the increased macrophage and leukocyte infiltration, and elevated inflammation are all more common in vulnerable, symptomatic plaques. Our results also showed a correlation between serum resistin levels and blood leukocytes, and a positive association of higher serum and plaque resistin 
levels with unstable plaque phenotypes, especially the ones containing higher grades of inflammatory infiltration, macrophage foamy cell formation, and lipid accumulation. However, the association of circulatory resistin levels with carotid artery diseases is still controversial. Young et al have reported that circulatory levels of resistin in elderly patients with T2DM gradually increase during the CAS progression from mild to severe disease form [Yang 2018]. Several recent studies also showed that circulatory resistin levels positively correlate with an increase in the carotid intima-media thickness (CIMT) as an early marker of carotid atherosclerosis [Rubio-Guerra 2013; Yazıc1 2012; Shin 2008; Robati 2014]. Contrary to this, Wang et al reported no correlation between circulatory resistin levels with the mean CIMT or its association with the presence of carotid atherosclerotic plaque [Wang 2014]. Likewise, Kunnari et al also showed no association between serum resistin levels and the mean CIMT [Kunnari 2006]. However, similar to our results they confirmed the association of circulatory resistin levels with blood leukocytes [Kunnari 2006].

Our study has several limitations. First, the relatively small sample size of patients recruited from a single medical center prevents us from generalizing the results. Second, the source of resistin in our study was not investigated, and third we measured total resistin and not its different multimers, which might have differing activities.

\section{Conclusion}

To the best of our knowledge this study was the first to assess the relationship of circulating and plaque resistin levels with ischemic stroke events and carotid plaque instability as evaluated by standard AHA plaque classification and semi-quantitative histological evaluations. Our results suggest that the serum resistin levels may be a clinical marker of plaque vulnerability and ischemic stroke events in moderate-to-high CAS patients, which highlights the possible relationship between plaque resistin expression and histological features of plaque vulnerability. Future large prospective studies are needed to confirm these results, and elucidate pathological mechanisms of plaque resistin expression on plaque vulnerability and the development of ischemic stroke symptomatology.

\section{REFERENCES}

Calabro P, Samudio I, Willerson JT, Yeh ET. 2004. Resistin promotes smooth muscle cell proliferation through activation of extracellular signal-regulated kinase 1/2 and phosphatidylinositol 3-kinase pathways. Circulation 110:3335-40.

Cho Y, Lee SE, Lee HC, et al. 2011. Adipokine resistin is a key player to modulate monocytes, endothelial cells, and smooth muscle cells, leading to progression of atherosclerosis in rabbit carotid artery. J Am Coll Cardiol 57:99-109.

Efstathiou SP, Tsiakou AG, Tsioulos DI, et al. 2007. Prognostic significance of serum resistin levels in patients with atherothrombotic ischemic stroke. Clin Chim Acta 378:78-85.

Faxon DP, Fuster V, Libby P, et al. 2004. for the American Heart Association. Atherosclerotic Vascular Disease Conference: Writing Group III: pathophysiology. Circulation 109:2617-25.
Flaherty ML, Kissela B, Khoury JC, et al. 2013. Carotid artery stenosis as a cause of stroke. Neuroepidemiology 40:36-41.

Gasbarrino K, Mantzoros C, Gorgui J, et al. 2016. Circulating chemerin is associated with carotid plaque instability, whereas resistin is related to cerebrovascular symptomatology. Arterioscler Thromb Vasc Biol 36:1670-8

Grant EG, Benson CB, Moneta GL, et al. 2003. Carotid artery stenosis: gray-scale and Doppler US diagnosis: Society of Radiologists in Ultrasound Consensus Conference. Radiology 229:340-6.

Jung HS, Park KH, Cho YM, et al. 2006. Resistin is secreted from macrophages in atheromas and promotes atherosclerosis. Cardiovasc Res 69:76-85.

Kunnari A, Ukkola O, Päivänsalo M, Kesäniemi YA. 2006. High serum resistin level is associated with enhanced highly sensitive C-reactive protein and leukocytes. J Clin Endocrinol Metab 91:2755-60.

Lovett JK, Gallagher PJ, Rothwell PM. 2004. Reproducibility of histological assessment of carotid plaque: Implications for studies of carotid imaging. Cerebrovasc Dis 18:117-23.

Lovett JK, Gallagher PJ, Hands LJ, Walton J, Rothwell PM. 2004. Histological correlates of carotid plaque surface morphology on lumen contrast imaging. Circulation 110:2190-7.

Mukherjee D, Yadav JS. 2000. Carotid and cerebrovascular disease. Cardiol Rev 8:322-32.

Naghavi M, Libby P, Falk E, et al. 2003. From vulnerable plaque to vulnerable patient: a call for new definitions and risk assessment strategies: Part I. Circulation 108:1664-72.

Nighoghossian N, Derex L, Douek P. 2005. The vulnerable carotid artery plaque: current imaging methods and new perspectives. Stroke 36:2764-72.

Ntaios G, Gatselis NK, Makaritsis K, Dalekos GN. 2013. Adipokines as mediators of endothelial function and atherosclerosis. Atherosclerosis 227:216-21.

Park HK, Ahima RS. 2013. Resistin in rodents and humans. Diabetes Metab J 37:404-14.

Perovic E, Mrdjen A, Harapin M, Tesija KA, Simundic AM. 2017. Diagnostic and prognostic role of resistin and copeptin in acute ischemic stroke. Top Stroke Rehabil 24:614-18.

Prugger C, Luc G, Haas B, Arveiler D, et al. 2012. PRIME Study Group Adipocytokines and the risk of ischemic stroke: the PRIME Study. Ann Neurol 71:478-86.

Prugger C, Luc G, Haas B, et al. 2013. PRIME Study Group. Multiple biomarkers for the prediction of ischemic stroke: the PRIME study. Arterioscler Thromb Vasc Biol 33:659-66.

Robati RM, Partovi-Kia M, Haghighatkhah HR, Younespour S, Abdollahimajd F. 2014. Increased serum leptin and resistin levels and increased carotid intima-media wall thickness in patients with psoriasis: is psoriasis associated with atherosclerosis? J Am Acad Dermatol 71:642-8.

Rubio-Guerra AF, Cabrera-Miranda LJ, Vargas-Robles H, MacedaSerrano A, Lozano-Nuevo JJ, Escalante-Acosta BA. 2013. Correlation between levels of circulating adipokines and adiponectin/resistin index with carotid intima-media thickness in hypertensive type 2 diabetic patients. Cardiology 125:150-3.

Shin HJ, Park S, Yoon SJ, et al. 2008. Association between serum resistin and carotid intima media thickness in hypertension patients. Int J Cardiol 125:79-84. 
Stary HC. 2000. Natural history and histological classification of atherosclerotic lesions: An update. Arterioscler Thromb Vasc Biol 20:1177-8.

Verma S, Li SH, Wang CH, et al. 2003. Resistin promotes endothelial cell activation: further evidence of adipokine-endothelial interaction. Circulation 108:736-40.

von Reutern GM1, Goertler MW, Bornstein NM, et al. 2012. Neurosonology Research Group of the World Federation of Neurology. Grading carotid stenosis using ultrasonic methods. Stroke 43:916-21.

Wang H, Wang YT, Fan L, et al. 2014. Resistin might not be a risk factor for carotid artery atherosclerosis in elderly Chinese males. J Geriatr
Cardiol 11:222-8.

Xu W, Yu L, Zhou W, Luo M. 2006. Resistin increases lipid accumulation and CD36 expression in human macrophages. Biochem Biophys Res Commun 351:376-82.

Yang W, Li Y, Wang JY, Han R, Wang L. 2018. Circulating levels of adipose tissue-derived inflammatory factors in elderly diabetes patients with carotid atherosclerosis: a retrospective study. Cardiovasc Diabetol 17:75.

Yazıcı D, Yavuz D, Öğünç AV, et al. 2012. Serum adipokine levels in type 1 diabetic patients: association with carotid intima media thickness. Metab Syndr Relat Disord 10:26-31. 